\title{
A influência das relações e o uso de álcool por adolescentes*
}

\author{
Miria Benincasa ${ }^{1}$ \\ Ana Luiza Tavares ${ }^{1}$ \\ Vivian Miucha Moura Barbosa ${ }^{1}$ \\ Mayara de Paula Lajara ${ }^{1}$ \\ Manuel Morgado Rezende ${ }^{1}$ \\ Maria Geralda Viana Heleno ${ }^{1}$ \\ Eda Marconi Custódio ${ }^{1}$
}

O álcool é a substância cada vez mais presente no consumo de adolescentes. O presente estudo teve como objetivo descrever sobre uso de álcool e a qualidade das relações sociais entre familiares, amigos e identificar como estas influenciam no consumo de álcool. Trata-se de um estudo transversal cujos dados coletados foram com uma amostra de 2423 estudantes, matriculados no Ensino Médio. Os dados expressam a significante presença do álcool nas relações e a ligação de uso com as relações tanto familiares como sociais de pares, independente da qualidade percebida nessas relações. Os dados são relevantes para se pensar na efetividade das intervenções de políticas públicas considerando os vínculos familiares e sociais.

Descritores: Adolescência; Álcool; Família; Estudantes; Grupos.

\footnotetext{
* Artigo extraído de tese de doutorado "Avaliação da qualidade de vida e uso de drogas em adolescentes do Municipio de São Paulo", apresentada ao Instituto de Psicologia, Universidade de São Paulo, SP, Brasil.

${ }^{1}$ Universidade Metodista de São Paulo, São Bernardo do Campo, SP, Brasil.
} 


\title{
The influence of relationships and alcohol use by adolescents
}

Alcohol is a substance that is increasingly present in adolescent consumption. The present study aimed to describe the use of alcohol and the quality of social relations between family and friends to identify how they influence alcohol consumption. It is a cross-sectional study, whose data were collected with a sample of 2423 students, enrolled in High School. The data expresses the significant presence of alcohol in the relationships and the use link with both family and social relationships of peers, regardless of the quality perceived in these relationships. The data are relevant for thinking about the effectiveness of public policy interventions considering family and social ties.

Descriptors: Adolescence; Alcohol; Family; Students; Groups.

\section{La influencia de las relaciones y el uso de alcohol por los adolescentes}

\begin{abstract}
El alcohol es la sustancia cada vez más presente en el consumo de este estudio fue describir adolescentes. O sobre el consumo de alcohol y la calidad de las relaciones sociales entre los miembros de la familia y amigos para determinar cómo estas influyen en el consumo de alcohol. Trata es un estudio transversal cuyos datos se recogieron con una muestra de 2423 estudiantes matriculados en la escuela secundaria. Os datos expresan la importante presencia de alcohol en las relaciones y el uso de la conexión con las relaciones tanto familiares y sociales parejas, independientemente de las relajones. El dato es relevante para pensar acerca de la efectividad de las intervenciones de política pública, considerando los lazos familiares y sociales.
\end{abstract}

Descriptores: Adolescencia; Alcohol; La Familia; Estudiantes; Grupos.

\section{Introdução}

A adolescência é caracterizada pela fase da vida em que ocorrem as mudanças mais significativas ao desenvolvimento. A exposição a fatores de risco nessa fase traz a necessidade de ampliar os conhecimentos sobre fatores influentes e protetivos a esses adolescentes.

Segundo os autores ${ }^{(1)}$, a adolescência é vista como uma fase de maior vulnerabilidade, contribuindo para que o jovem se coloque em situações de risco e aumente a probabilidade da experimentação de drogas. Atualmente, de acordo com a Organização Mundial da Saúde (OMS), o consumo de substâncias psicoativas (SPA) por adolescentes tem sido um dos mais prevalentes problemas de saúde mental(2).

Estudos nacionais e internacionais afirmam que o álcool é de fácil propagação, refletindo no consumo precoce e exagerado. Evidentemente, este é um fator de risco para o desenvolvimento de estilos de consumo que levem à dependência e suas complicações. Em levantamento realizado em 2010 com estudantes do ensino fundamental e médio, indica-se que o álcool é a substância mais consumida entre adolescentes, com início aos 13 anos de idade ${ }^{(3)}$.

Os efeitos agudos do consumo, como alegria e loquacidade, são mascaradores da ação inibidora do álcool sobre o sistema nervoso, assim como os anestésicos. O álcool age indiretamente sobre o sistema límbico, que tem um papel crucial na expressão das emoções e na atividade do sistema de recompensa do cérebro, área ventral e nucleus accumbens ${ }^{(4)}$.

O uso do álcool entre adolescentes é um tema que levanta bastante controvérsia no meio social e no âmbito acadêmico brasileiro. Ao mesmo tempo em que é ilegal a venda para menores de 18 anos, sua ingestão acaba sendo aceita e praticada por esses jovens, seja no ambiente domiciliar, em festividades ou em ambientes públicos. Existe uma aceitação social para o consumo que é exposto pelas propagandas ${ }^{(5)}$. 
Entre as questões levantadas por estudos, encontram-se afirmações sobre a influência dos pares e grupos no consumo, considerando que tanto os tidos bons relacionamentos como os ruins são fatores de influência para o consumo(6-10). A questão da precocidade e da oferta estar atribuída a laços afetivos também é outro fator indicado para influenciar no estilo de consumo desses adolescentes ${ }^{(1,11-12)}$

O consumo de bebidas alcoólicas é tido como comportamento socialmente aceito e, segundo as pesquisas, quando esse comportamento está incorporado nas relações familiares, sendo o consumo permitido ou não, emerge outro fator de risco para o desenvolvimento de estilo de consumo que desencadeie a questão da dependência(13-16).

\section{Material e Método}

Trata-se de um estudo transversal cujos dados foram coletados em 2010, entre alunos matriculados no Ensino Médio nas escolas do município de São Paulo, sobre uso de álcool e a qualidade das relações sociais entre familiares e amigos. Este estudo foi realizado com uma amostra de 2423 estudantes, sendo que 1059 são do sexo masculino (45,8\%) e do feminino 1254 (54,2\%). Em relação à idade dos participantes, não possuem diferença significativa, sendo que a amostra para ambos os sexos se apresenta igual no comparativo de idades.

Os dados foram coletados por meio de um questionário de identificação geral dos adolescentes e outro questionário visando à questão do uso de drogas por adolescentes. No questionário de identificação geral, solicitou-se os seguintes dados: nome, idade, sexo, série, duas questões propostas pela ABEP (2007) para classificação econômica no Brasil e informações gerais de saúde. A apresentação das questões propostas pela ABEP em 2007 (Critério padrão de classificação econômica Brasil / 2008) segue o modelo adotado pelo CEBRID em seu VI Levantamento Nacional sobre o Consumo de Drogas Psicotrópicas entre Estudantes do Ensino Fundamental e Médio das Redes Pública e Privada de Ensino nas 27 Capitais Brasileiras.

O outro questionário, sobre o uso substâncias psicoativas, incluindo o uso de álcool, foi proposto pela Organização Mundial da Saúde (OMS) e desenvolvido pela WHO - Reserch and Reporting Project on the Epidemiology of Drug Dependence, o qual identifica o consumo e frequência do uso de álcool e outras substâncias e também questiona sobre a percepção das relações familiares. Tal questionário também foi utilizado no VI Levantamento Nacional sobre o Consumo de Drogas Psicotrópicas entre Estudantes do Ensino
Fundamental e Médio das Redes Pública e Privada de Ensino nas 27 Capitais Brasileiras.

\section{Resultados e Discussão}

Conforme descrito, os dados foram coletados em 2010, entre alunos matriculados no Ensino Médio nas escolas no município de São Paulo, e partem de pesquisa sobre qualidade de vida e uso de drogas, tendo como amostra 2434 participantes, sendo 1059 do sexo masculino (45,8\%) e do feminino $1254(54,2 \%)$. As idades dos participantes não apresentaram diferença significativa, sendo assim a amostra quando olhada para ambos os sexos se apresenta igual no comparativo de idades.

Pesquisas internacionais recentes demonstram uma constante de que nas amostras de adolescentes menores de 18 anos o álcool já foi experimentado ou já faz parte de um consumo que pode ser de menor frequência até um uso abusivo ${ }^{(1,6-7,10,14,16)}$.

Em levantamento de dados nacionais e internacionais sobre o consumo de álcool, consta que entre adolescentes de 14 a 16 anos o álcool é a substância mais consumida e esse uso excessivo ou abusivo em idade precoce frequentemente é associado como um fator de risco para a dependência na vida adulta ${ }^{(1,3,8-9)}$.

Nos dados levantados pela presente pesquisa, na amostra, $82,1 \%$ afirmam já ter feito uso de alguma bebida alcoólica, $71 \%$ afirmaram que no presente ano da pesquisa já haviam feito uso de álcool e no último mês $25,6 \%$ afirmaram ter consumido um copo ou menos. Da comparação entre os dados emerge a realidade do quanto o álcool está presente no consumo dos adolescentes.

Em pesquisa com 83 participantes na Alemanha, a maioria da amostra de adolescentes com idade entre 16 e 18 anos afirma ter comportamento de beber em excesso. Dessa mesma amostra, $32 \%$ afirmam ter esse comportamento ao menos duas vezes no mês e $28 \%$ afirmam ter esse comportamento de três a quatro vezes no mês( ${ }^{(6)}$. Outra amostra descreve que entre adolescentes portugueses da mesma faixa etária a afirmação de beber em excesso é de $22 \%{ }^{(11)}$.

$\mathrm{Na}$ amostra da presente pesquisa, sobre o comportamento de beber em excesso, $63 \%$ afirmam não ter tido tal comportamento de consumo. Os demais $32,5 \%$ afirmam já ter tido comportamento de beber em excesso. Sobre o mesmo comportamento, no último mês, a amostra indica que 81,9\% afirmam não ter tido esse comportamento recente. As demais respostam indicam que $5,4 \%$ dos participantes afirmam ter bebido em excesso ao ponto da embriaguez há menos de uma 
semana, 3,6\% há menos de 15 dias e 4,3\% há vinte dias ou mais.

Apesar de destoar em números significativamente $e$ ter a distinção de tamanho da amostra, comparando com os levantamentos internacionais ${ }^{(6,8,11)}$, o comportamento de beber em excesso ou se embriagar está presente entre os adolescentes e com ocorrência maior a uma vez por mês.

Questões culturais e de legislação devem ser consideradas nas comparações entre as amostras e os dados apresentados pelas pesquisas. A legislação brasileira não permite a comercialização de bebidas alcoólicas para menores de 18 anos assim como em outros países da América Latina, tal regulamentação também ocorre em países como Noruega. Na Europa, em alguns lugares, a legislação permite o consumo a partir dos 16 anos; nos EUA, a venda legal é somente aos 21 anos ${ }^{(1,6,11,16)}$. Contudo, a constante é que o álcool faz parte em algum momento das experimentações desses adolescentes.

As variadas formas de legalização do álcool e o persistente consumo independente dessas formas sugerem que existem outras variáveis que devem ser consideradas nas estratégias de intervenção ao consumo excessivo de álcool por esses adolescentes.

Dessas variáveis é a influência dos pares e família, o primeiro local de consumo identificado em pesquisas é o ambiente familiar e casa de amigos, existindo a distinção dessa afirmação para o gênero feminino, em que o primeiro consumo tende a ocorrer em locais públicos. Sobre isso, identifica-se que a questão de papéis de gênero pode ser uma influência, uma vez que o consumo de álcool por homens é mais aceito que o consumo por mulheres ${ }^{(11)}$.

Sobre o primeiro contato com o álcool, 30,4\% dos participantes afirmaram que amigos ofereceram pela primeira vez, dentre estes representam entre as meninas $17,4 \%$. Já entre os meninos, a oferta feita pelos amigos representa $14,5 \%$ (Tabela 1 ).

No entanto, o presente estudo identificou que $24,8 \%$ dos participantes afirmaram que veio de um familiar a oferta de bebidas alcoólicas; entre estes, 11,8\% são adolescentes do sexo masculino e $14,4 \%$ adolescentes do sexo feminino, ou seja, a primeira oferta pelo meio familiar torna-se representativa para o gênero feminino, destoando da afirmação de aceitação familiar do beber atribuída ao gênero masculino.

Sobre onde estavam esses adolescentes quando consumiram álcool pela primeira vez, $26,6 \%$ responderam que estavam em casa; destes, $17,5 \%$ eram meninas e $10,4 \%$ meninos; $21,6 \%$ responderam que estavam na casa de amigos, sendo que $11,1 \%$ dos que responderam eram meninos e $11,5 \%$ meninas. Indicando que o ambiente familiar e suas relações entram como uma influência para estimular o primeiro contato com álcool de forma significativa entre o gênero feminino.

Nesses dados, percebe-se que os responsáveis pela primeira oferta de bebida e o local em que ocorreu o primeiro contato para essa amostra são por meio dos laços sociais e familiares. Corroborando para o que apontam pesquisas internacionais ${ }^{(1,6-7,10-11,16)}$, o primeiro contato com a bebida ocorre por meio dos laços familiares e o primeiro episódio de embriaguez ocorre fora do conhecimento dos pais, junto com amigos em festas, mas no caso das meninas a família e o ambiente familiar surgem como primeiro meio de contato.

Esse consumo no meio familiar explicita que adolescentes que afirmaram ter sido expostos a comportamento de consumo de álcool na infância possuíam tendência a apresentar consumo de alto risco na adolescência ${ }^{(16)}$. E a precocidade da exposição é acompanhada pela afirmação de que esse primeiro contato ocorreu por meio de algum familiar próximo ${ }^{(1,11)}$.

A questão da aceitação do consumo e oferta de consumo estar presente nas relações sociais mais afetivas e familiares apresenta-se como uma variável de grande relevância a ser considerada quando o consumo excessivo de álcool por adolescentes tornase um risco(1,6,10-11,16).

Corroborando com a ideia de que o consumo aceito é uma das influências para a manutenção do comportamento, os dados da presente pesquisa apontam que dos adolescentes da amostra afirmam que o consumo de álcool tende a ocorrer significativamente em bares e baladas (25\%), na casa de amigos (19,8\%) e em casa $(15,4 \%)$.

Tabela 1: Relação entre gênero e quem ofereceu bebida pela primeira vez. São Paulo, SP, Brasil, 2010

\begin{tabular}{cccccccc}
\hline \multirow{2}{*}{ Nunca Bebi } & \multicolumn{5}{c}{ Quem lhe ofereceu bebida alcoólica pela primeira vez? } \\
\cline { 2 - 8 } & & Familiares & Amigos & $\begin{array}{c}\text { Comprou } \\
\text { Sozinho }\end{array}$ & Outros & Não Lembro \\
\cline { 3 - 8 } Masc. & $\mathrm{n}^{\circ}$ & 181 & 272 & 335 & 107 & 25 & 139 \\
& $\%$ & $7,8 \%$ & $11,8 \%$ & $14,5 \%$ & $4,6 \%$ & $1,1 \%$ & $6,0 \%$ \\
Fem. & $\mathrm{n}^{\circ}$ & 162 & 332 & 403 & 76 & 34 & 247 \\
& $\%$ & $7,0 \%$ & $14,4 \%$ & $17,4 \%$ & $3,3 \%$ & $1,5 \%$ & $10,7 \%$ \\
\hline
\end{tabular}


Tabela 2: Relação entre companhia em consumo e gênero. São Paulo, SP, Brasil, 2010.

\begin{tabular}{ccccccc}
\hline & & Não Costumo & Familiares & Amigos & Sozinho & Outros \\
\hline \multirow{2}{*}{ Masc. } & $\mathrm{n}^{\circ}$ & 401 & 168 & 463 & 23 & 5 \\
& $\%$ & $17,3 \%$ & $7,3 \%$ & $20,0 \%$ & $1,0 \%$ & $0,2 \%$ \\
Fem. & $\mathrm{n}^{\circ}$ & 399 & 262 & 564 & 17 & 15 \\
& $\%$ & $17,2 \%$ & $11,3 \%$ & $24,3 \%$ & $0,7 \%$ & $0,6 \%$ \\
\hline
\end{tabular}

Os adolescentes afirmam que encontram como razões para o consumo de álcool estar em grupo de pares, aceitação social, evitar ou modificar emoções negativas e o ambiente no qual estão inseridos ${ }^{(6,11)}$.

A percepção de um suporte dos amigos alto ou percepção de boas relações com os amigos surge como um determinante para o aumento das chances de embriaguez nos grupos de adolescentes ${ }^{(10)}$.

No presente trabalho, amigos são apontados como as principais companhias durante o consumo de álcool. Na amostra, $24,3 \%$ das participantes do sexo feminino afirmaram que bebem com amigos e 11,3\% responderam que fazem o consumo de álcool junto com familiares. Entre os participantes do sexo masculino, $20 \%$ afirmam que costumam consumir álcool com os amigos e $7,3 \%$ na companhia de familiares (Tabela 2 ).

O comportamento de beber nos grupos de amigos também é indicado com maior incidência de ocorrência nas pesquisas internacionais pela questão da aceitação social a tal comportamento ${ }^{(6-7,10-11)}$.

A influência do consumo em grupos surge também naqueles grupos que são descritos como positivos e amistosos pelos adolescentes, a exemplo o consumo de álcool entre adolescente é maior entre aqueles que praticam esportes coletivos, do que aqueles que praticam atividades esportivas individuais, implicando aí uma variável significativa: a influência dos grupos tidos como positivos no comportamento de beber(6).

Dentre os fatores discutidos para a melhor compreensão do comportamento de consumo de álcool por adolescentes, encontram-se citadas nas pesquisas como fatores correlacionados a aceitação social, a influência dos pares e a influência da família, mas se faz necessário refletir sobre o quanto essa aceitação social não participa de uma construção familiar. Os dados apresentam o primeiro contato pela via familiar, ofertado por um membro da família ou pelos próprios pais.

A porcentagem da amostra da presente pesquisa afirma existir o consumo com os pais, mas sendo que se apresenta em menor porcentagem quando em comparação com os dados internacionais ( $11 \%$ das meninas afirmam e 7,3\% dos meninos). Ainda assim, os dados apontam que o consumir é aceito e permitido pela família e para o gênero feminino isso ocorre em maior porcentagem comparando com o gênero masculino, tido como gênero a ter o consumo como melhor aceito.

Adolescentes que afirmam um consumo frequente de álcool com os pais também afirmam em altas proporções episódios de alto consumo de álcool $(48,4 \%)$ e embriaguez recorrente (35.4\%), em comparação com aqueles que afirmavam não fazer consumo de álcool com os pais $(15.5 \% \text { e } 7.1 \%)^{(16)}$.

Sendo relevante evidenciar que o consumo permissivo de álcool na família é uma das influências a serem consideradas para a correlação entre o consumo frequente e abusivo do álcool entre adolescentes. A família se encontra como transmissora de valores, padrões de comportamento, tradições e a forma como o funcionamento familiar aceita e lida com o consumo de álcool terá reflexo na percepção dos adolescentes sobre o consumo também ${ }^{(15)}$.

Indicadores em pesquisas internacionais possibilitam apontar relações entre o consumo de álcool e as relações familiares. As variações das relações familiares e das percepções destas podem ser influências para um consumo abusivo do álcool, assim como podem ser indicadores de um consumo consciente ${ }^{(12)}$.

Quando questionados sobre a percepção da relação entre os pais, na amostra, a maioria respondeu que percebe como bom (30,4\% masculino e $31,8 \%$ feminino). A distinção maior ocorreu entra na resposta de pais separados, representando $10,9 \%$ nas adolescentes e $6,9 \%$ nos adolescentes.

Buscou-se compreender se ocorreria a existência de correlação entre a percepção da relação dos pais com o uso em excesso, porém a presente amostra não foi possível indicar essa relação, o que os dados apontam é que $62,5 \%$ daqueles que responderam fazer um consumo frequente de álcool afirmam perceber que os pais têm um bom relacionamento entre eles. Outro dado é que, da amostra que afirma fazer consumo frequente de álcool, 19,4\% têm pais separados.

A qualidade da percepção da relação entre adolescentes e pais, nas pesquisas internacionais, surge como variável a ser considerada para a existência ou não de um comportamento abusivo no uso de álcool. Outra variável considerada nas pesquisas internacionais e que revelou apreciável associação com as variações do comportamento de uso de álcool foi considerar a percepção dos pais sobre o consumo e se no meio familiar existiam 
controle e monitoramento do uso de álcool(1,6,10,13,15-16). Na presente pesquisa, esses aspectos não foram levantados, o que sugere a necessidade de aprofundamento para melhor compreensão da associação entre percepção parental com o uso de álcool e se de fato a correlação existe.

Contudo, a constante entre a presente pesquisa e os demais trabalhos internacionais sugere que a família é considerável influência, quando desponta com expressividade no primeiro contato com o álcool e quando afirma a norma social de aceitação do comportamento de consumir bebidas alcoólicas.

O funcionamento familiar além de influência para o estilo de consumo também surge como variável da resiliência para lidar com os problemas que advêm de um consumo excessivo. Um funcionamento familiar considerado bom, no qual ocorre o consumo excessivo, teria então melhores condições de lidar e fornecer apoio às questões de advêm do uso exagerado do álcool por adolescentes $^{(15)}$.

Ou seja, apesar de não expressar correlação explícita entre a percepção da relação entre os pais com o comportamento de uso moderado ou excessivo do álcool existe uma influência da família para o desenvolvimento do estilo de consumo. Considerando que na presente amostra tanto para a primeira experimentação como o ambiente para consumo e companhia de consumo a família é citada, inclina-se, portanto, para a reflexão de que se faz necessário considerar também o consumo de álcool e a frequência deste pelos pais e a percepção destes sobre o consumo dos filhos para melhor compreender esse espaço familiar que pode tanto ser protetivo como um fator de risco para o desenvolvimento da dependência e os demais agravos do consumo excessivo de álcool por adolescentes.

\section{Considerações finais}

A adolescência é considerada um período de especial preocupação quanto a questões do uso de substâncias. Os riscos do uso excessivo à saúde e desenvolvimento de dependências nos adolescentes colocam-se perante as políticas públicas de saúde e sociais em busca de intervenções que entendam os fatores associados a esse consumo.

Diante disso, a presente pesquisa buscou, ao se debruçar sobre as questões de qualidade de vida e uso de substâncias, em foco o consumo de álcool, ampliar o conhecimento sobre os fatores de influência para o comportamento de consumo e consumo excessivo pelos adolescentes e fatores relacionados.

Dentre os dados que emergem da amostra participante, chama atenção o fato de que o primeiro contato com o álcool e que a companhia citada para o consumo entre as meninas adolescentes seja a família, pontada de forma significativa. Essa informação remete à reflexão de como as questões de gênero dentro das relações familiares estariam influenciando no estilo de consumo de álcool. A família ao permitir e aceitar que esse consumo de álcool ocorra e assim também o estimulando para que seja feito na companhia da mesma, estaria então afirmando que esse beber ao ocorrer sob o olhar dos pais teria um fator protetivo ou estaria indicando que existe uma equiparação na aceitação do comportamento de beber tanto para o gênero feminino como para o masculino? E sob esses questionamentos como isso influenciaria no consumo das adolescentes? Seria este um indicador para a maior compreensão do porquê do aumento do consumo de álcool por mulheres? Esses questionamentos demonstram a necessidade de ser ampliar as investigações e trabalhos que tragam à tona os fatores de consumo e consumo de risco considerando as questões de gênero.

Os dados que indicam a presença da família no primeiro contato com álcool também colocam em foco que a percepção de boas relações associadas ao consumo de álcool pelos pais pode corroborar para que o beber seja aceito e tido como norma social e assim influenciando no estilo de consumo desses adolescentes, conforme inclinam-se as pesquisas internacionais e nacionais a afirmar.

Entende-se que para se pensar melhores intervenções com adolescentes quando a temática é o uso de substância, faz-se necessário compreender as influências que interferem nos estilos de consumo, uma vez que somente as proibições legais não inibem o uso, muito menos o uso excessivo, tendo em vista que essa problemática é tida por países com diferenciadas idades mínimas para o consumo. É preciso considerar as questões culturais que estão atreladas e a própria propagação da aceitação de uso pelo meio social e familiar, aproximando, assim, os serviços de saúde dos núcleos de convivência desses adolescentes, como família, escola e lazer.

\section{Referências}

1. Williams AV, Meyer E, Pechansky F. Desenvolvimento de um Jogo Terapêutico para Prevenção de Recaída e Motivação para Mudança em Jovens Usuários de Drogas. Psicologia: Teor Pesq. 2007; 23(4):407-14.

2. Organização Mundial Da Saúde. DSM-IV: Manual Diagnóstico e Estatístico de Transtornos Mentais. 4. ed. Porto Alegre: Artes Médicas; 1995.

3. Carlini ELA, Noto AR, Sanchez, ZVDM, Carlini CMA, Locatelli DP, Abeid LR. VI Levantamento Nacional sobre 
o Consumo de Drogas Psicotrópicas entre Estudantes do Ensino Fundamental e Médio das Redes Pública e Privada de Ensino nas 27 Capitais Brasileiras - 2010. São Paulo: CEBRID - Centro Brasileiro de Informações sobre Drogas; 2011.

4. Menezes SM. Neuroanatomia aplicada. Rio de Janeiro: Guanabara Koogan; 1999;

5. Padilha MI, Silva SE. Atitudes e comportamentos de adolescentes em relação à ingestão de bebidas alcoólicas. Rev Esc Enferm USP. 2011;45:1063-9.

6. Jander A, Mercken L, Crutzen R, Vries H. Determinants of binge drinking in a permissive environment: focus grous interviews with Dutch adolescents and parents. BMC Public Health. 2013;13:882.

7. Liu XC, Keyes KM, Li G. Work stress and alcohol consumption among adolescents: moderation by family and peer influences. BMC Public Health. 2014;14:1303.

8. Rozin L, Zagonel IP. Fatores de risco para dependência de álcool em adolescentes. Acta Paul Enferm. 2011; 25:314-8. 9. Thora MK, Francisco R, Antonia JI, Carmen M. Perceived Quality of Social Relations and Frequent Drunkenness: A Cross-Sectional Study of Spanish Adolescents. Alcohol Alcoholism. 2014;49(4):466-71.

10. Trujillo AM, Flórez IA. Consumo de Alcohol en lós Adolescentes de Chía y su Percepción del Consumo y de la Permisividad Parental frente al Uso de Sustancias. Rev Colombiana Psicol. 2013; 22(1):41-57.

11. Brito I, Precioso JAG, Correia C, Albuquerque C, Samorinha C, Cunha-Filho H, Becoña E. Fatores associados ao consumo de álcool na adolescência, em função do gênero. Psicol Saúde Doenças. 2015;16(3):392-410.

12.Cordova D, Heinze J, Mistry R, Hsieh HF, Stoddard S, Wright CPS, Zimmerman MA. Family Functioning and Parent Support Trajectories and Substance Use and Misuse among Minority Urban Adolescents: A Latent Class Growth Analysis. Subst Use Misuse. 2014; 49(14):1908-19.

13. Abar CC, Jackson KM, Colby SM, Barnett NP. Parentchild discrepancies in reports of parental monitoring and their relationship to adolescent alcohol-related behaviors. J Youth Adolesc. 2015; 44(9):1688-701.

14. Amaral MTR, Carvalho RC, Costa MCO, Cruz ANL, Lopes TC, Matos AM. Uso frequente e precoce de bebidas alcoólicas na adolescência: análise de fatores associados. Adolesc Saúde. 2013;10: 25-32.

15. Cortés G, García M, Rivera AS. Potencial resiliente em familias con adolescentes que consumen y no consumen alcohol. Acta Colombiana Psicol. 2015;18(2):163- 72.

16. Pape H, Rossow I, Storvoll EE. Is Drinking with Parents Associated with High-Risk Drinking among Adolescents? European Addiction Research. Eur Addict Res. 2015;21:291-9 